\title{
Optical and electronic properties of silver nanoparticles embedded in cerium oxide
}

Jacopo Stefano Pelli Cresi, ${ }^{1,2}$ Enrico Silvagni, ${ }^{1}$ Giovanni Bertoni,,${ }^{2,3}$ Maria Chiara Spadaro, ${ }^{1,2}$ Stefania Benedetti, ${ }^{2}$ Sergio Valeri, ${ }^{1,2}$ Sergio D’Addato, ${ }^{1,2}$ Paola Luches ${ }^{2}$

${ }^{1}$ Dipartimento di Scienze Fisiche Informatiche e Matematiche, Università degli Studi di Modena e Reggio Emilia, Via G. Campi 213/a, 41125 Modena, Italy

${ }^{2}$ Istituto Nanoscienze, Consiglio Nazionale delle Ricerche, Via G. Campi 213/a, 41125 Modena, Italy

${ }^{3}$ Istituto Materiali per l'Elettronica e il Magnetismo, Consiglio Nazionale delle Ricerche, Parco Area delle Scienze 37/a, Parma, Italy.

\begin{abstract}
Wide band gap oxides can be sensitized to visible light by coupling them with plasmonic nanoparticles. We investigate the optical and electronic properties of composite materials made of Ag nanoparticles (NP) embedded within cerium oxide layers of different thickness. The electronic properties of the materials are investigated by x-ray and ultraviolet photoemission spectroscopy, which demonstrate the occurrence of static charge transfers between the metal and the oxide and its dependence on the NP size. UV-Vis spectrophotometry measurements show that the materials have a strong absorption in the visible range induced by the excitation of localized surface plasmon resonances. The plasmonic absorption band can be modified in shape and intensity by properly tuning the NP size and density.
\end{abstract}

\section{Introduction}

In recent years, research activities on active catalysts for environmental protection have gained an increased relevance and urgency, also due to the widespread concern for global warming. Cerium oxide based catalysts are active in a number of important environmental reactions, the most successful commercial applications being in car catalytic converters and in antiparticulate filters for diesel engines. ${ }^{1}$ More recent applications of cerium oxide based materials include fuel cell electrodes ${ }^{2,3}$ and catalysts for the synthesis of solar fuels. ${ }^{4}$ However, the application of cerium oxide based materials as photocatalysts is limited by the $\mathrm{CeO}_{2}$ band gap in the ultraviolet (3.2-3.6 eV) ${ }^{5}$ and by the relatively low electron mobility of the material. ${ }^{6}$ Methods to sensitize cerium oxide based materials to visible light include nanostructuration, ${ }^{7-9}$ defect engineering, ${ }^{10}$ doping ${ }^{11,12}$ and the coupling with metallic nanoparticles (NPs), like for example Au or Ag. ${ }^{9,11,13-15}$ In the latter case visible light excites the socalled localized surface plasmon resonances (LSPR) within the NPs. The energy of such resonant excitations can be transferred to the neighboring oxide modifying its properties and its catalytic activity. ${ }^{13,15}$ The interaction between the NPs and the oxide after LSPR excitation may involve different mechanisms. Direct energy transfers as well as direct or indirect charge transfer processes may occurr. ${ }^{16-18}$ Indeed, the specific processes which can take place and their efficiency depend on various characteristics of the investigated materials, like for example the dielectric environment of the NPs, their size, shape and density, the atomic scale structure of the interfaces, the alignment of the energy bands of the oxide and of the metal NP and the Schottky barrier which is formed between the two materials. The need for a design-driven synthesis of efficient photocatalytic materials calls for accurate studies of the involved materials in terms of the above mentioned parameters. 
Static charge transfer processes, ${ }^{19-22}$ as well as more complex interaction mechanisms, ${ }^{23-25}$ have been identified on model systems made of metal NP supported on cerium oxide surfaces. The influence of such processes on the functionality of the composite materials has been recognized, although the resulting optical properties are comparatively less understood.

Among the investigated metal NPs, Ag has the advantage of having a strong absorption cross section in the visible range and of being relatively less critical than for example Au or Pt in terms of availability and cost. Composite systems made of Ag NPs embedded within cerium oxide matrixes have recently been investigated and a long-living and efficient plasmon-mediated charge transfer has been hypothesized, based on the results of fast transient absorption spectroscopy. ${ }^{26}$ Moreover, a superior LSPR-induced catalytic activity of $\mathrm{Ag} / \mathrm{CeO}_{2}$ systems under visible light irradiation was recently demonstrated. ${ }^{11,27}$

Metals deposited by physical methods on oxide surfaces typically self-assemble into nanoparticles, with size and shape determined by the metal-oxide interaction, by the oxide surface morphology and by the deposition temperature. ${ }^{28}$ In most cases both the NP size and/or their density tend to increase with the amount of deposited metal. Here we present a systematic study of the electronic and optical properties of Ag NPs of different size and density coupled with cerium oxide. The investigation provides important insights into the interaction between the two materials, important for a knowledgedriven design of cerium oxide based materials with a high efficiency towards visible light absorption.

\section{Experimental}

The samples investigated in this study are Ag NPs of variable size and density embedded within or supported on $\mathrm{CeO}_{2}$ films. Double side polished $\mathrm{MgO}(001)$ single crystals were chosen as supports for the $\mathrm{Ag} \mathrm{NPs}-\mathrm{CeO}_{2}$ systems, since they ensure a good optical transparency in the visible range. The substrates were cleaned by two subsequent ultrasonic baths in warm $(373 \mathrm{~K})$ acetone and in isopropanol for 15 minutes, and introduced in an ultra-high vacuum apparatus for further cleaning by heating at $673 \mathrm{~K}$ for $30 \mathrm{~min}$. Cerium oxide films of different thickness were grown by reactive molecular beam epitaxy (MBE), by evaporating $\mathrm{Ce}$, using an electron beam cell, in an oxygen partial pressure of $1 \times 10^{-7}$ mbar on the $\mathrm{MgO}$ substrate kept at room temperature $(\mathrm{RT})$. The Ag NPs were selfassembled on the cerium oxide film surface at RT by depositing Ag from a Knudsen cell. Both the Ce and the Ag evaporators were calibrated by a quartz crystal microbalance. The size and density of the Ag NPs obtained depend on the amount of Ag deposited. We have focused the present study on NPs formed by depositing $2 \AA, 4 \AA$ and $12 \AA$ of $\mathrm{Ag}$ on the cerium oxide film surface, where the nominal Ag amount refers to the equivalent thickness of a uniform Ag layer fully covering the oxide surface. The samples for ex-situ characterization were covered by a further cerium oxide layer, to protect the $\mathrm{Ag}$ NPs from contamination induced by air exposure. To increase the optical absorption of the $2 \AA$ and $4 \AA$ Ag NPs sample, we grew multiple layers of Ag NPs, separated by $\mathrm{CeO}_{2}$ films, as schematically shown in Figure 1. For the case of $4 \AA \mathrm{Ag}$ NPs three different samples with NP layers separated by oxide layers of $0.5 \mathrm{~nm} 1 \mathrm{~nm}$ and $2 \mathrm{~nm}$ thickness were prepared, in order to investigate also the effect of the dielectric thickness on the optical properties (see Figure 1). Pure $\mathrm{CeO}_{2}$ films of different thickness without Ag NPs were also grown and measured for comparison. 


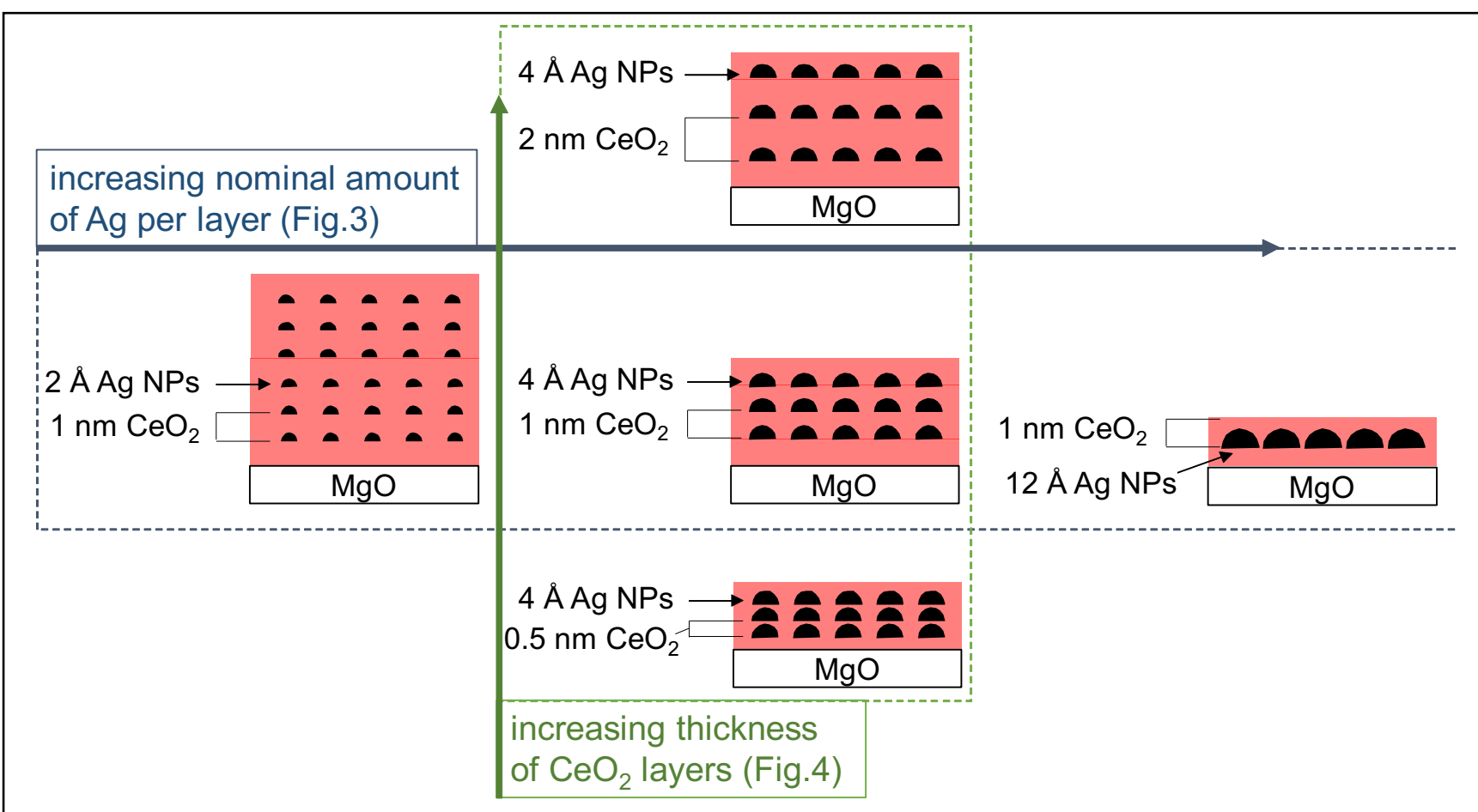

Figure 1: Schematic cross sections of the samples investigated by UV-Vis spectrophotometry.

The electronic properties of the samples were analyzed in-situ by X-ray photoemission spectroscopy (XPS) and ultraviolet photoemission spectroscopy (UPS). For the XPS measurements we used Al K $\alpha$ photons from a double anode X-ray source and a hemispherical electron analyzer in normal emission geometry. The analysis of Ce $3 \mathrm{~d}$ spectra is a powerful method to precisely determine the evolution of stoichiometry of the surface of cerium oxide based materials. The fitting of the spectra was performed using a procedure, introduced by Romeo et al. ${ }^{29}$ and further improved by Skala and coworkers ${ }^{30}$, which we have already used to evaluate the $\mathrm{Ce}^{3+}$ and $\mathrm{Ce}^{4+}$ concentration in a number of previous studies ${ }^{31,32}$. Briefly, it consists in fitting the spectra using five Voigt-shaped doublets, three related to photoemission from $\mathrm{Ce}^{4+}$ ions and two related to photoemission from $\mathrm{Ce}^{4+}$ ions with different final states. The procedure is relatively precise in detecting changes in the concentration of $\mathrm{Ce}^{3+}$ and $\mathrm{Ce}^{4+}$ in different samples, while the accuracy on the numbers obtained is affected by a relatively large error. The UPS measurements were acquired using a helium lamp and a hemispherical electron analyzer at normal emission.

The optical properties were investigated ex-situ using a UV-Vis spectrophotometer equipped with a xenon lamp, a grating monochromator and a silicon photodetector (detection range 250-750 nm). The incidence angle between the incident photon beam and the sample normal was set to $22^{\circ}$. The absorbance A was evaluated by measuring the transmittance $\mathrm{T}$ and the reflectance $\mathrm{R}$ as $\mathrm{A}=1-\mathrm{T}-\mathrm{R}$.

The morphology of the system was also investigated ex-situ by scanning transmission electron microscopy (STEM), using a JOEL JEM-2200FS microscope located at CNR-IMEM in Parma, on a sample grown directly on a TEM grid (consisting in thin carbon films supported on a $\mathrm{Cu}$ mesh) with the same procedures used for the samples grown on $\mathrm{MgO}$. The TEM was operated also with an energy dispersive X-ray spectrometer (EDXS), to determine the distribution of Ag and Ce on the sample surface. 


\section{Results and discussion}

The morphology of the MBE-grown Ag NPs embedded between two $\mathrm{CeO}_{2}$ layers was investigated by TEM. Figure 2 a reports a representative annular dark-field scanning TEM (ADF-STEM) image of a portion of a $4 \AA$ Ag NP sample embedded between two cerium oxide layers of $1 \mathrm{~nm}$ thickness (Figure 2 a). The Ag particles appear bright in the image due to the higher average atomic number (about 1.5 times the $\mathrm{CeO}_{2}$ value by averaging in the unit cell volume). The Ag size distribution, shown in Figure 2 $\mathrm{b}$, was measured from a series of images (using conventional particle analysis as implemented in the Image J software ${ }^{33}$ ). The size distribution is rather broad, it has a maximum around $3 \mathrm{~nm}$, and the maximum NP diameter is approximately $10 \mathrm{~nm}$. EDXS maps from the Ce-L edge (cyan) and Ag-L edge (magenta), shown in Figure $2 \mathrm{c}$, confirm that Ag is concentrated into NPs, while cerium is

a)

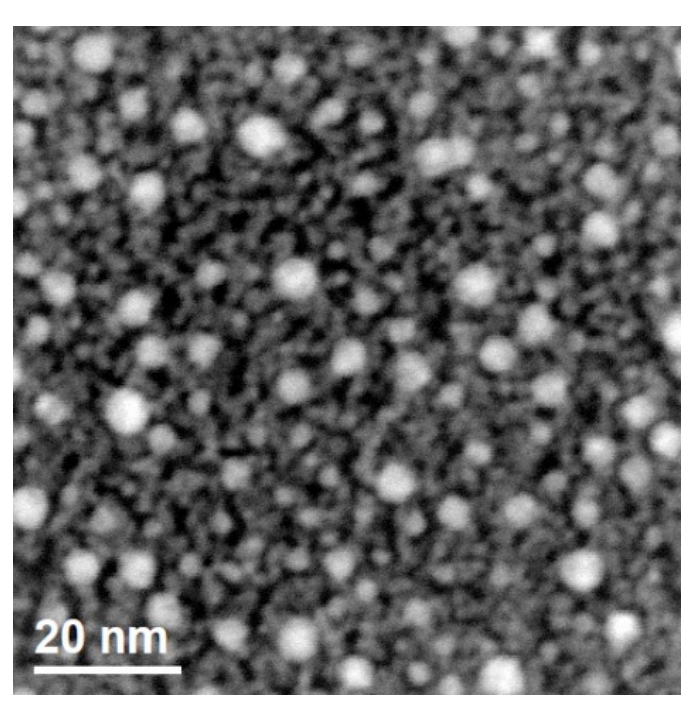

C)

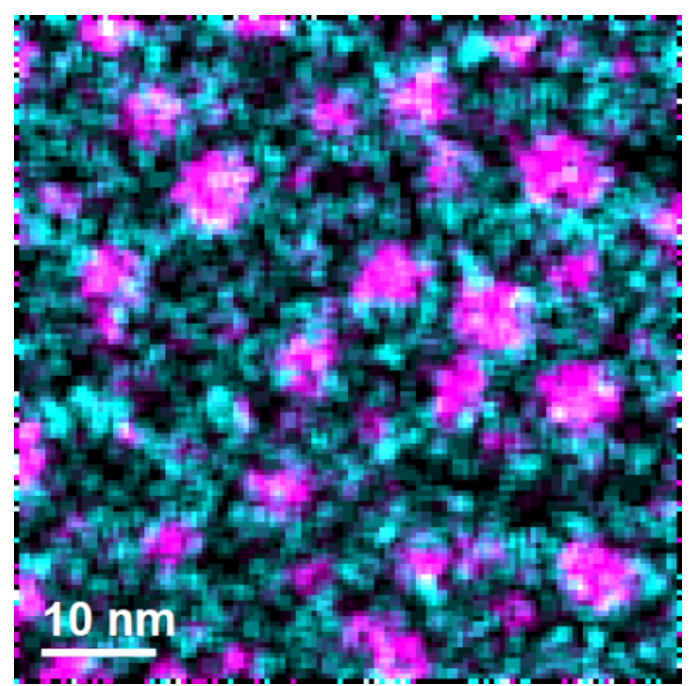

b)

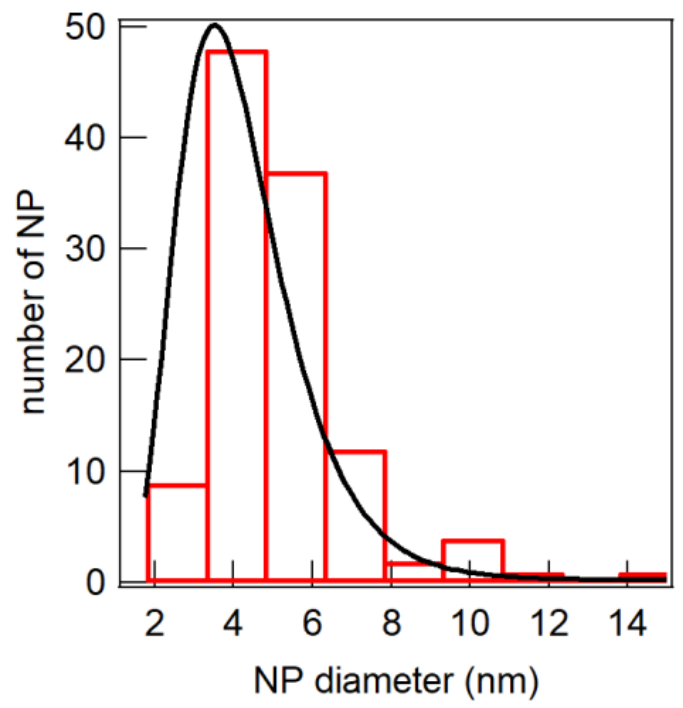

d)

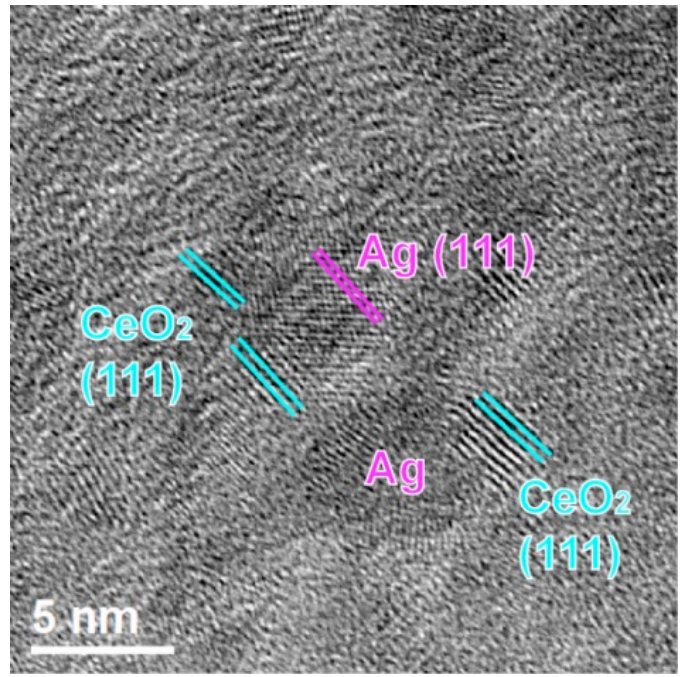

Figure 2: a) STEM image of a TEM of a $4 \AA$ Ag sample: b) size distribution extracted from STEM images; c) EDXS map of Ce L edge intensity (cyan) and Ag L edge (magenta) intensity; d) HRTEM in which some of the $\mathrm{Ag}(111)$ and $\mathrm{CeO}_{2}(111)$ planes are evidenced. 
uniformly distributed on the sample surface. Figure $2 \mathrm{~d}$ reports a high-resolution TEM (HRTEM) image acquired on the same sample, which demonstrates that most of the Ag nanoparticles have a polyhedral shape and a polycrystalline structure, like on similar systems [S. D’Addato, V. Grillo, A. di Bona, P. Luches, S. Frabboni, S. Valeri, P. Lupo, F. Casoli, F. Albertini, Nanotechnology 24 (2013) 495703]. Cerium oxide forms small crystalline domains with a lateral size of a few $\mathrm{nm}$. In some cases, the oxide grains show an epitaxial relation with the metal NPs surface. This is evidenced in Figure $2 \mathrm{~d}$, which highlights $\mathrm{CeO}_{2}(111)$ planes $\left(d_{l}=3.12 \AA\right)$ aligned parallel to $\mathrm{Ag}(111)$ planes $\left(d_{2}=2.36 \AA\right)$. Although the in-plane lattice mismatch in the $\mathrm{CeO}_{2}(111)$ and $\mathrm{Ag}(111)$ planes is rather large, it can be hypothesized that, as on many other fcc-metal (111) surfaces, cerium oxide adopts its most stable (111) orientation also on $\operatorname{Ag}(111)$ surfaces through the formation of an in-plane coincidence lattice. ${ }^{34-36}$

Cerium oxide films deposited on polished $\mathrm{MgO}(001)$ surfaces and on TEM grids with the same procedures are expected to have a comparable roughness, since polished oxide surfaces are not atomically flat and no simple epitaxial relation is expected to be established between the $\mathrm{MgO}(001)$ surface and the most stable low-index cerium oxide planes. Therefore, we expect the Ag NP morphology and structure to be comparable in the samples deposited on the two different substrates.

The size and the shape of the NPs, and in particular their aspect ratio, defined as the ratio between the NP diameter and its height, determine the energy of the optical absorption features ${ }^{37}$. Indeed, also the dielectric in which the NPs are embedded plays a relevant role in determining the polarizability and hence the dielectric response of the material. Moreover, the plasmonic resonance modes can be shifted by interparticle interactions.[mettici il libro di Stefania senza esplicitare la paagina] We investigated the dependence of the optical response on the above mentioned parameters in the case of the $\mathrm{Ag} \mathrm{NPs}_{\mathrm{SeO}} \mathrm{CeO}_{2}$ system, by measuring the UV-Vis absorbance spectra as a function of Ag nominal thickness and as a function of the thickness of the $\mathrm{CeO}_{2}$ film in which the $\mathrm{Ag}$ NPs are embedded. Figure 3 a shows the $\mathrm{UV}-\mathrm{Vis}$ absorbance spectra of $2 \AA, 4 \AA, 12 \AA \mathrm{Ag}$ samples embedded within two ceria layers of a constant thickness of $1 \mathrm{~nm}$. The $2 \AA$ and $4 \AA$ Ag samples consist of six and three repeated layers of $\mathrm{Ag}$ NPs, respectively (see Figure 1). The spectra in Figure 3 a show a peak at approximately $275 \mathrm{~nm}$,

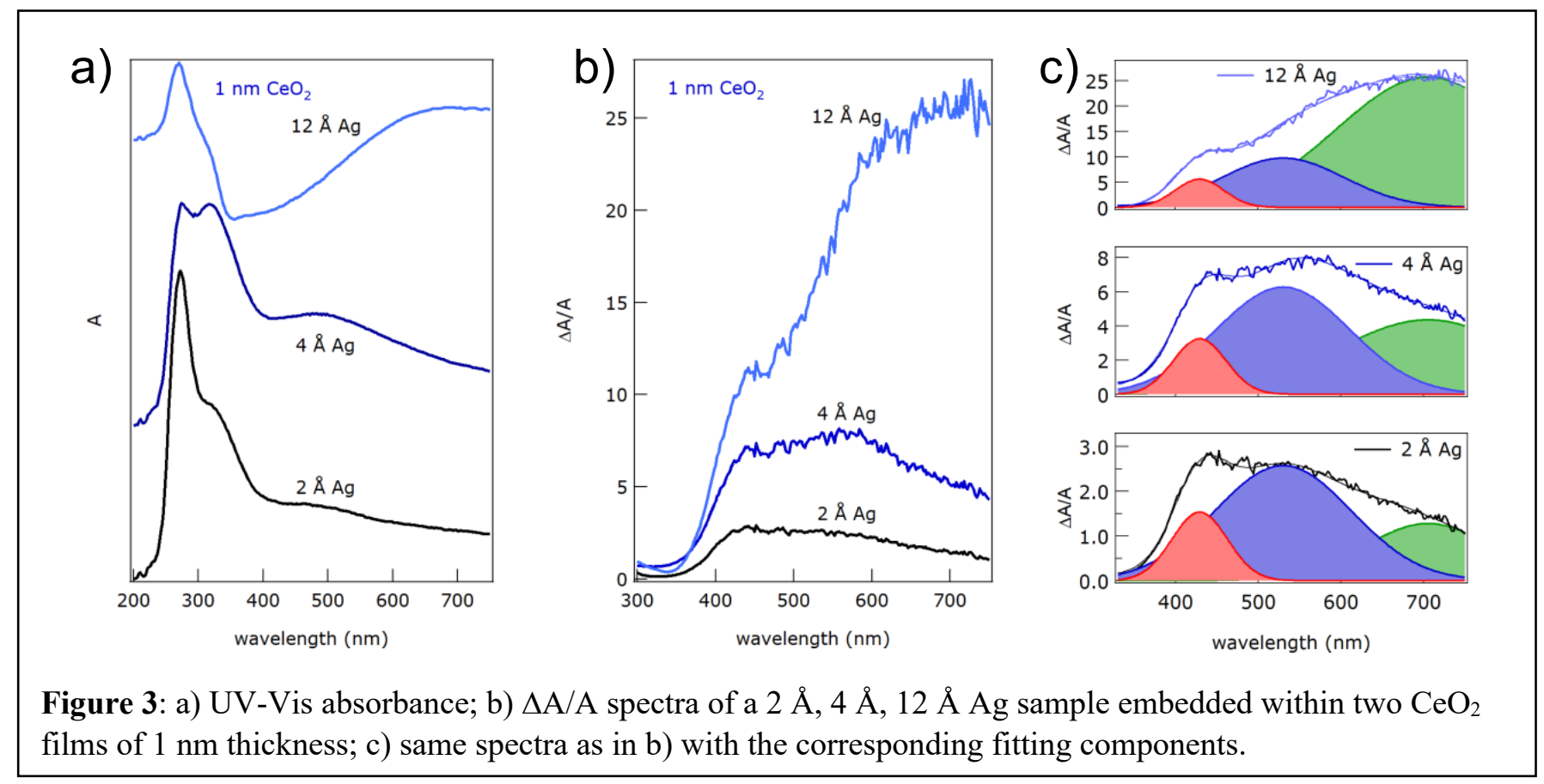


corresponding to the excitation of interband transitions in the $\mathrm{MgO}$ substrate, a second peak at approximately $320 \mathrm{~nm}$, corresponding to interband transitions in the $\mathrm{CeO}_{2}$ matrix, and a broad LSPR band in the visible range (the $\mathrm{UV}-\mathrm{V}$ is spectra of bare $\mathrm{MgO}$ and $\mathrm{CeO}_{2} / \mathrm{MgO}$ are shown in the Supporting Material). In order to compare the LSPRs-related features in the different samples, we considered the differential absorbance spectra $\frac{\Delta A}{A}=\frac{A-A_{\mathrm{CeO}_{2}}}{A_{\mathrm{CeO}_{2}}}$, where $A_{\mathrm{CeO}_{2}}$ is the absorbance of a $\mathrm{CeO}_{2}$ film without embedded Ag NPs. The resulting spectra, reported in Figure $3 \mathrm{~b}$, show an increasing intensity as the amount of Ag per layer is increased, although the total amount of Ag is nominally the same in each sample. This is not surprising since the absorption cross section is proportional to the volume of the NPs, when the light wavelength is much larger than the NP size as in the case here investigated. The broad absorption feature in the visible range is composed by different peaks, one centered slightly above $400 \mathrm{~nm}$, whose position appears unaltered in the different samples, and a second very broad feature at higher wavelengths, which significantly increases in intensity and progressively shifts towards red as the amount of Ag per layer is increased. The morphology of the samples here investigated is quite complex, with relatively broad distributions of sizes and interparticle distances (Figure 2). In spite of this, to understand the evolution of the optical absorbance as a function of the amount of Ag deposited within each NP layer, the situation was simplified by fitting of the absorbance spectra with three Gaussians, as shown in Figure 3 c (see also Supporting Material for details). The fitting procedure used merely aims at pointing out the dominant contributions to the optical absorption. The first Gaussian peak, centered at $429 \mathrm{~nm}$, can be ascribed to the excitation of transverse dipolar oscillations in the Ag NPs perpendicular to the sample surface. The second peak, centered at $530 \mathrm{~nm}$, can be ascribed to an excitation of the LSPR in the longitudinal direction, i.e. parallel to the surface. The third peak, centered at $700 \mathrm{~nm}$, can be ascribed to longitudinal LSPR, modified by the interaction among NPs, when their mutual distance is smaller than approximately 1 $\mathrm{nm}$. The intensity of the latter peak in fact increases as a function of the nominal Ag thickness, i.e. with increasing Ag NP density. We expect that in the samples with denser and larger Ag NPs, the fraction of 
NPs, which are close enough that their longitudinal LSPR feature is shifted towards red, increases. Simulations of the extinction coefficient, reported in the Supporting Material, confirm the given assignment. The broadness of the experimental absorbance features accounts for the size distribution and for other electronic effects which will be discussed further on.

To have more information on the optical properties of $\mathrm{Ag}$ NPs coupled with $\mathrm{CeO}_{2}$, we also investigated the evolution of the absorbance spectra in three samples with a fixed amount of Ag NPs per layer ( $4 \AA$ ) separated by $\mathrm{CeO}_{2}$ layers of $0.5 \mathrm{~nm}, 1 \mathrm{~nm}$ and $2 \mathrm{~nm}$ thickness, respectively (see Figure 1). The three samples consist of three repetitions of Ag NP layers. The UV-Vis spectra and the corresponding $\frac{\Delta A}{A}$ spectra are reported in Figure 4. In Figure 4 a we can clearly see that the feature corresponding to $\mathrm{CeO}_{2}$ interband transitions at $320 \mathrm{~nm}$ has a progressively increasing intensity as compared to the LSPR feature in the visible, as the thickness of the $\mathrm{CeO}_{2}$ layers is increased. The $\frac{\Delta A}{A}$ spectra (Figure $4 \mathrm{~b}$ ) show very broad LSPR-related absorption features, which were fit using three Gaussians centered in the same positions as the ones used for the fitting of the spectra of Figure 3, as shown in Figure $4 \mathrm{c}$. The weight of the third peak, corresponding to resonances modified by interparticle interactions, with respect to the first two peaks corresponding to dipolar oscillations within individual nanoparticles, increases with decreasing thickness of the $\mathrm{CeO}_{2}$ film which separates the $\mathrm{Ag}$ NP layers, confirming the given assignment. In the different samples in fact the density of NPs in the out of plane direction is expected to decrease with increasing $\mathrm{CeO}_{2}$ layer thickness, while the in-plane density of NPs is the same (Figure 1), in agreement with a slightly but progressively decreasing intensity of the feature related to interacting NPs.
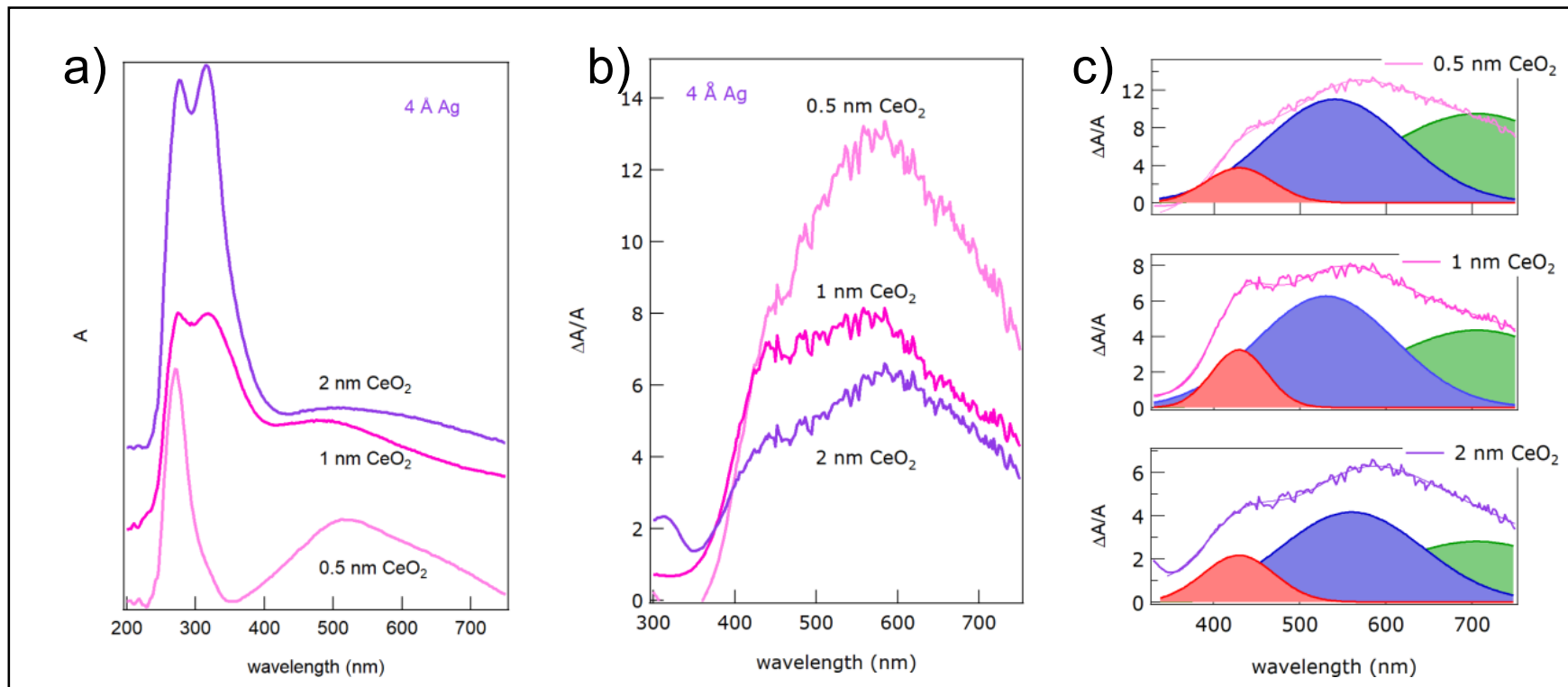

Figure 4: a) UV-Vis absorbance and b) $\Delta \mathrm{A} / \mathrm{A}$ spectra of a $4 \AA \mathrm{Ag}$ samples embedded within $\mathrm{CeO}_{2}$ layers of $0.5 \mathrm{~nm}, 1 \mathrm{~nm}$ and $2 \mathrm{~nm}$ thickness; c) same spectra as in b) with the corresponding fitting components. 

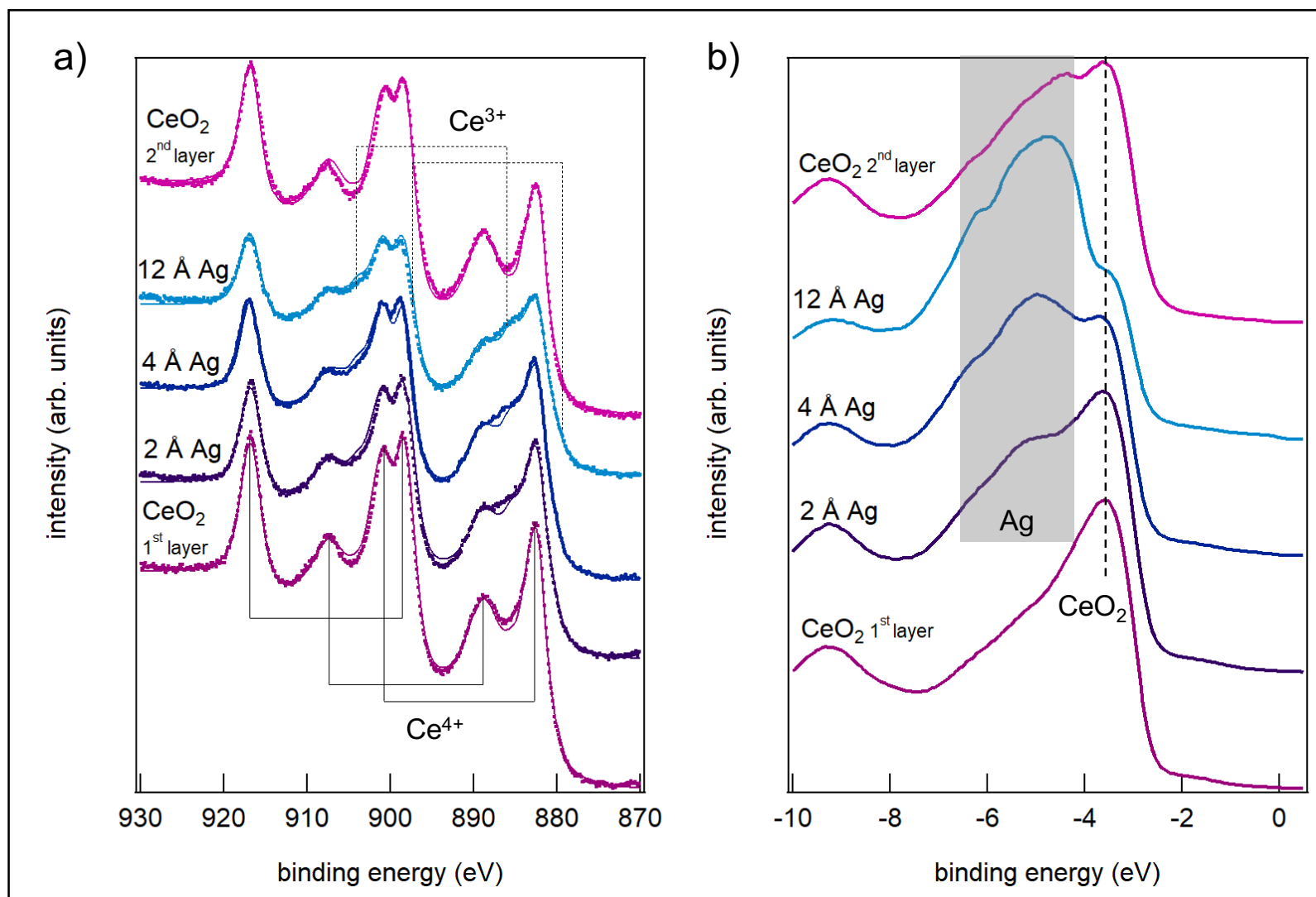

Figure 5: a) XPS Ce 3d and b) UPS spectra acquired in-situ at the different stages of the growth of a 12 $\AA \mathrm{Ag}$ sample between two $\mathrm{CeO}_{2}$ layers of $1 \mathrm{~nm}$ thickness.

In-situ XPS/UPS analysis offers the possibility of investigating the electronic properties of the samples at the different stages of the growth, and to obtain a more detailed understanding of the interaction between $\mathrm{Ag}$ NPs and $\mathrm{CeO}_{2}$, interesting for a clearer understanding of the optical response. Figure 5 shows the XPS Ce $3 \mathrm{~d}$ and UPS spectra acquired at different stages of the growth of a $12 \AA \mathrm{Ag}$ sample embedded between two $\mathrm{CeO}_{2}$ layers of $1 \mathrm{~nm}$ thickness. After the deposition of the first $\mathrm{CeO}_{2}$ layer, the $\mathrm{Ce} 3 \mathrm{~d}$ spectrum (Figure $5 \mathrm{a}$ ) has the characteristic shape of Ce ions mainly in the 4+ oxidation state. The fitting of the spectra with $\mathrm{Ce}^{3+}$ - and $\mathrm{Ce}^{4+}$-related components as outlined in the experimental section indicates the additional presence of a minor, though non-negligible, concentration of $\mathrm{Ce}^{3+}$ ions (Table 1), which are probably located at defect sites. These are formed due to the limited mobility of the adatoms during the growth, carried out at RT on the magnesium oxide substrate. After the growth of $2 \AA$ of $\mathrm{Ag}$ on the $\mathrm{CeO}_{2}$ film, the $\mathrm{Ce} 3 \mathrm{~d}$ XPS spectra show the appearance of features at $884 \mathrm{eV}$ and $904 \mathrm{eV}$, ascribed to the most intense $\mathrm{Ce}^{3+}$ related doublet. The weight of such features progressively increases with the amount of deposited $\mathrm{Ag}$, indicating an increasing concentration of $\mathrm{Ce}^{3+}$ ions. A dominant $\mathrm{CeO}_{2}$ surface stoichiometry is restored after the growth of the topmost cerium oxide layer. The $\mathrm{Ce}^{3+}$ concentration after each step of the growth, obtained by fitting the Ce $3 \mathrm{~d}$ XPS spectra, is reported in Table 1. A progressive reduction of comparable entity as the one observed here was already measured on epitaxial $\mathrm{CeO}_{2}$ films grown on $\mathrm{Pt}(111)$ after the growth of $\mathrm{Ag} \mathrm{NPs},{ }^{20}$ and it was ascribed to a charge transfer from the NPs to the substrate, an effect often altering the properties of small nanoparticles coupled with oxides. ${ }^{19}$ 
Previous works demonstrated small shifts of the LSPR with NP charging. ${ }^{38,39}$ Although for the samples here investigated it is quite difficult to quantify the expected shifts, we can hypothesize that the broadness of the observed plasmonic absorption features in the spectra of Figures 3 and 4, may be ascribed not only to the relatively broad NP size distribution (see Figure $1 \mathrm{~b}$ ), but also to the different charge densities, which in turn depend on the NP size and on the nucleation site on the oxide surface. Moreover, modifications of the local polarizability of the oxide are expected to be induced by the charge transfer process.

The UPS spectrum of the $\mathrm{CeO}_{2}$ film (Figure $5 \mathrm{~b}$, bottom spectrum) shows a dominant broad feature between 2 and $7 \mathrm{eV}$, which is due to emission from the valence band of $\mathrm{O} 2 \mathrm{p}$ character. The feature progressively changes shape with increasing amount of $\mathrm{Ag}$ deposited, due to the appearance of features related to the $\mathrm{Ag}$ valence band at binding energies around $5 \mathrm{eV}$ [Al momento non trovo niente di meglio che questo: Paola Finetti, Luisa Ferrari, Sergio D’Addato, Surface Science 677 (2018) 213] and to the attenuation of the $\mathrm{CeO}_{2}$-related main feature around $3 \mathrm{eV}$. After the growth of the topmost $1 \mathrm{~nm}$ thick $\mathrm{CeO}_{2}$ layer (Figure $5 \mathrm{~b}$, topmost spectrum) the Ag-related features are still visible, demonstrating that the coverage of the Ag NPs is possibly not uniformly as thick as $1 \mathrm{~nm}$, as expected given the size of the Ag NPs and the relatively rough surface morphology.

\begin{tabular}{|c|c|}
\hline sample & $\mathrm{c}_{\mathrm{Ce} 3+}(\mathbf{\%})$ \\
\hline $1 \mathrm{~nm} \mathrm{CeO}_{2}$ & $11 \pm 2$ \\
\hline $1 \mathrm{~nm} \mathrm{CeO}_{2}+2 \AA \mathrm{Ag}$ & $20 \pm 2$ \\
\hline $1 \mathrm{~nm} \mathrm{CeO}_{2}+4 \AA \mathrm{Ag}$ & $23 \pm 2$ \\
\hline $1 \mathrm{~nm} \mathrm{CeO} 2+12 \AA \mathrm{Ag}$ & $26 \pm 2$ \\
\hline $1 \mathrm{~nm} \mathrm{CeO}_{2}+12 \AA \mathrm{Ag}+1 \mathrm{~nm} \mathrm{CeO}_{2}$ & $9 \pm 2$ \\
\hline
\end{tabular}

Table 1: $\mathrm{Ce}^{3+}$ concentration evaluated from the fitting of Ce $3 \mathrm{~d}$ XPS spectra

\section{Conclusions}

We show that Ag deposited by MBE on cerium oxide films with a non-ordered surface spontaneously aggregates into particles of nanometric size. If embedded within two cerium oxide films, the Ag NPs show an LSPR-related optical absorption band in the visible range, with a shape which changes as the amount of $\mathrm{Ag}$ deposited and the thickness of the $\mathrm{CeO}_{2}$ dielectric are varied. Some charge transfer from the NPs to the oxide is observed and hypothesized to affect the optical response.

\section{Acknowledgements}

The present work is performed with the financial support of MIUR through PRIN project no. 2015CL3APH.

\section{References}

[1] T. Montini, M. Melchionna, M. Monai and P. Fornasiero, Chem Rev 116, 5987 (2016).

[2] V. Bambagioni, C. Bianchini, Y. Chen, J. Filippi, P. Fornasiero, M. Innocenti, A. Lavacchi, A. Marchionni, W. Oberhauser and F. Vizza, ChemSusChem 5, 1266 (2012).

[3] R. Fiala, A. Figueroba, A. Bruix, M. Vaclavu, A. Rednyk, I. Khalakhan, M. Vorokhta, J. Lavkova, F. Illas, V. Potin, I. Matolinova, K. M. Neyman and V. Matolin, Applied Catalysis B: Environmental 197, 262 (2016). 
[4] F. Lin, M. Rothensteiner, I. Alxneit, J. A. van Bokhoven and A. Wokaun, Energy \& Environmental Science 9, 2400 (2016).

[5] S. Guo, H. Arwin, S. N. Jacobsen, K. Järrendahl and U. Helmersson, Journal of Applied Physics 77, 5369 (1995).

[6] H. L. Tuller and A. S. Nowick, Journal of The Electrochemical Society 126, 209 (1979).

[7] A. Corma, P. Atienzar, H. García and J.-Y. Chane-Ching, Nat Mater 3, 394 (2004).

[8] Y. Li, Q. Sun, M. Kong, W. Shi, J. Huang, J. Tang and X. Zhao, The Journal of Physical Chemistry C 115, 14050 (2011).

[9] A. Primo, T. Marino, A. Corma, R. Molinari and H. García, J Am Chem Soc 133, 6930 (2011).

[10] M. M. Khan, S. A. Ansari, D. Pradhan, D. H. Han, J. Lee and M. H. Cho, Industrial \& Engineering Chemistry Research 53, 9754 (2014).

[11] M. Kazazi, B. Moradi and M. Delshad Chermahini, Journal of Materials Science: Materials in Electronics 30, 6116 (2019).

[12] L. Yue and X.-M. Zhang, Journal of Alloys and Compounds 475, 702 (2009).

[13] S. M. Kim, H. Lee, K. C. Goddeti, S. H. Kim and J. Y. Park, The Journal of Physical Chemistry C 119, 16020 (2015).

[14] A. Tanaka, K. Hashimoto and H. Kominami, J Am Chem Soc 134, 14526 (2012).

[15] B. Li, T. Gu, T. Ming, J. Wang, P. Wang, J. Wang and J. C. Yu, Acs Nano 8, 8152 (2014).

[16] N. Wu, Nanoscale 10, 2679 (2018).

[17] K. Wu, J. Chen, J. R. McBride and T. Lian, Science 349, 632 (2015).

[18] R. Long and O. V. Prezhdo, J Am Chem Soc 136, 4343 (2014).

[19] Y. Lykhach, S. M. Kozlov, T. Skála, A. Tovt, V. Stetsovych, N. Tsud, F. Dvořák, V. Johánek, A. Neitzel, J.

Mysliveček, S. Fabris, V. Matolín, K. M. Neyman and J. Libuda, Nat Mater 15, 284 (2015).

[20] P. Luches, F. Pagliuca, S. Valeri, F. Illas, G. Preda and G. Pacchioni, The Journal of Physical Chemistry C 116, 1122 (2012).

[21] A. Bruix, J. A. Rodriguez, P. J. Ramírez, S. D. Senanayake, J. Evans, J. B. Park, D. Stacchiola, P. Liu, J. Hrbek and F. Illas, J Am Chem Soc 134, 8968 (2012).

[22] T. E. James, S. L. Hemmingson, T. Ito and C. T. Campbell, The Journal of Physical Chemistry C 119, 17209 (2015).

[23] Y. Lykhach, A. Bruix, S. Fabris, V. Potin, I. Matolínová, V. Matolín, J. Libuda and K. M. Neyman, Catalysis Science \& Technology 7, 4315 (2017).

[24] J. A. Farmer and C. T. Campbell, Science 329, 933 (2010).

[25] F. Benedetti, P. Luches, M. C. Spadaro, G. Gasperi, S. D'Addato, S. Valeri and F. Boscherini, The Journal of Physical Chemistry C 119, 6024 (2015).

[26] J. S. Pelli Cresi, M. C. Spadaro, S. D'Addato, S. Valeri, S. Benedetti, A. Di Bona, D. Catone, L. Di Mario, P. O'Keeffe, A. Paladini, G. Bertoni and P. Luches, Nanoscale 11, 10282 (2019).

[27] P. Verma, Y. Kuwahara, K. Mori and H. Yamashita, Catal Today 324, 83 (2019).

[28] C. T. Campbell, Surface Science Reports 27, 1 (1997).

[29] M. Romeo, K. Bak, J. El Fallah, F. Le Normand and L. Hilaire, Surface and Interface Analysis 20, 508 (1993).

[30] T. Skála, F. Šutara, M. Škoda, K. C. Prince and V. Matolín, Journal of Physics: Condensed Matter 21, 055005 (2009).

[31] P. Luches, F. Pagliuca and S. Valeri, J Phys Chem C 115, 10718 (2011).

[32] P. Luches, F. Pagliuca and S. Valeri, Phys Chem Chem Phys 16, 18848 (2014).

[33] C. A. Schneider, W. S. Rasband and K. W. Eliceiri, Nature Methods 9, 671 (2012).

[34] P. Luches, L. Giordano, V. Grillo, G. C. Gazzadi, S. Prada, M. Campanini, G. Bertoni, C. Magen, F. Pagliuca, G. Pacchioni and S. Valeri, Adv Mater Interfaces 2, 1500375 (2015).

[35] S. Eck, C. Castellarin-Cudia, S. Surnev, M. G. Ramsey and F. P. Netzer, Surf Sci 520, 173 (2002).

[36] J. L. Lu, H. J. Gao, S. Shaikhutdinov and H. J. Freund, Surf Sci 600, 5004 (2006).

[37] I. Simonsen, R. Lazzari, J. Jupille and S. Roux, Phys Rev B 61, 7722 (2000). 
[38] M. Zapata Herrera, J. Aizpurua, A. K. Kazansky and A. G. Borisov, Langmuir 32, 2829 (2016).

[39] S. Benedetti, I. Valenti and S. Valeri, The Journal of Physical Chemistry C 123, 8206 (2019). 